\title{
Pseudo Central-Catheter Method for Asymmetrical Vaginal Prescription with Multi-Channel Cylindrical Applicators in Image-Guided High Dose-Rate Brachytherapy
}

\author{
Kelin Wang1* ${ }^{*}$ Michele Ferenci ${ }^{1}$, Kyle Padgett$^{2}$, Mutian Zhang ${ }^{3}$, Maria Irene Monterroso ${ }^{2}$, \\ Ming Chao ${ }^{4}$ \\ ${ }^{1}$ Department of Radiology, Pennsylvania State University, Hershey Medical Center, Hershey, USA \\ ${ }^{2}$ Department of Radiation Oncology, University of Miami, Miller School of Medicine, Miami, USA \\ ${ }^{3}$ Department of Radiation Oncology, University of Nebraska Medical Center, Omaha, USA \\ ${ }^{4}$ Department of Radiation Oncology, Icahn School of Medicine at Mount Sinai, New York, USA \\ Email: kwang1@hmc.psu.edu
}

Received 13 February 2015; accepted 4 April 2015; published 9 April 2015

Copyright (C) 2015 by authors and Scientific Research Publishing Inc.

This work is licensed under the Creative Commons Attribution International License (CC BY).

http://creativecommons.org/licenses/by/4.0/

c) (i) Open Access

\section{Abstract}

Multi-channel cylindrical applicators for high dose-rate (HDR) brachytherapy are utilized mainly for special gynecology cases often involving image-guidance high dose-rate (IG-HDR or IGBT) brachytherapy. In these cases, doses are prescribed to partial vaginal wall/depth where the disease is indicated by an MRI study scanned on the same day. These novel IG-HDR procedures are logistical challenges comparing to typical HDR out-patient treatments due to the complexity of planning for asymmetrical prescriptions as well as the extension of the whole procedure. Indeed the main advantages of HDR over LDR brachytherapy would be significantly weaken unless the IG-HDR procedures become more efficient in time, thus timely treatment planning becomes crucial. Based on our clinical experience, we propose the pseudo central-catheter method to reduce errors, to minimize uncertainty and to ensure efficiency. In this method, the central hollow path of the multi-channel cylindrical applicator is digitized into a pseudo catheter similar to other active catheters, and the asymmetrical prescription points could be generated corresponding to the pseudo dwelling points in the pseudo central-catheter. The pseudo dwelling points are later deleted after generating the prescription point arrays. This method is robust with minimal chance of errors or uncertainties, and demonstrates high efficiency with much less chance of uncertainty, which is significant for IG-HDR brachytherapy procedures utilizing multi-channel applicators.

${ }^{*}$ Corresponding author.

How to cite this paper: Wang, K., et al. (2015) Pseudo Central-Catheter Method for Asymmetrical Vaginal Prescription with Multi-Channel Cylindrical Applicators in Image-Guided High Dose-Rate Brachytherapy. International Journal of Medical Physics, Clinical Engineering and Radiation Oncology, 4, 124-131. http://dx.doi.org/10.4236/ijmpcero.2015.42016 


\section{Keywords}

\section{Pseudo Catheter, Asymmetrical Prescription, Multi-Channel Cylindrical Applicator}

\section{Introduction}

High dose-rate (HDR) brachytherapy with vaginal cylinder applicators are commonly performed for patients with early stage vaginal carcinomas, endometrial or cervical carcinomas. The typical treatment regions are either vaginal cuff or upper one-third of vagina, with prescription either to vaginal surface or a certain depth (e.g. 5 $\mathrm{mm}$ ) beyond the surface. However, in recent development of image-guided high dose-rate brachytherapy (IGHDR, or IGBT), an MRI scan is taken on the same day as the CT scan with the applicator in place, and the MRI scan is subsequently registered to the CT, thus the disease could be clearly contoured on the fused image set. The major advantage of IG-HDR brachytherapy is the healthy part of vaginal wall which could be possibly spared while treating the local disease. In these cases, the prescriptions are dose points only at the disease side of vaginal wall rather than to the whole cylindrical vaginal wall. Since doses are prescribed asymmetrically with respect to the center of cylindrical applicator, implementing a single-channel applicator is incapable to achieve this goal as since dose distribution is always symmetrical to the catheter, thus a multi-channel applicator must be implemented.

The idea on multi-channel cylindrical applicator for high dose-rate (HDR) brachytherapy was initially proposed by University of Miami [1] more than 2 decades ago, and was later adopted by vendors such as Nucletron $^{\mathrm{TM}}$, thus these applicators are often called a Miami Applicator. Prevalent multi-channel cylindrical applicator normally consists of a central channel (or a hollow path to allow a tandem applicator going through) with surrounding channels distributed at regular intervals outside of the central channel (Figure 1). In most situations, the HDR source will not dwell inside the central channel because there is no catheter; the source will only dwell

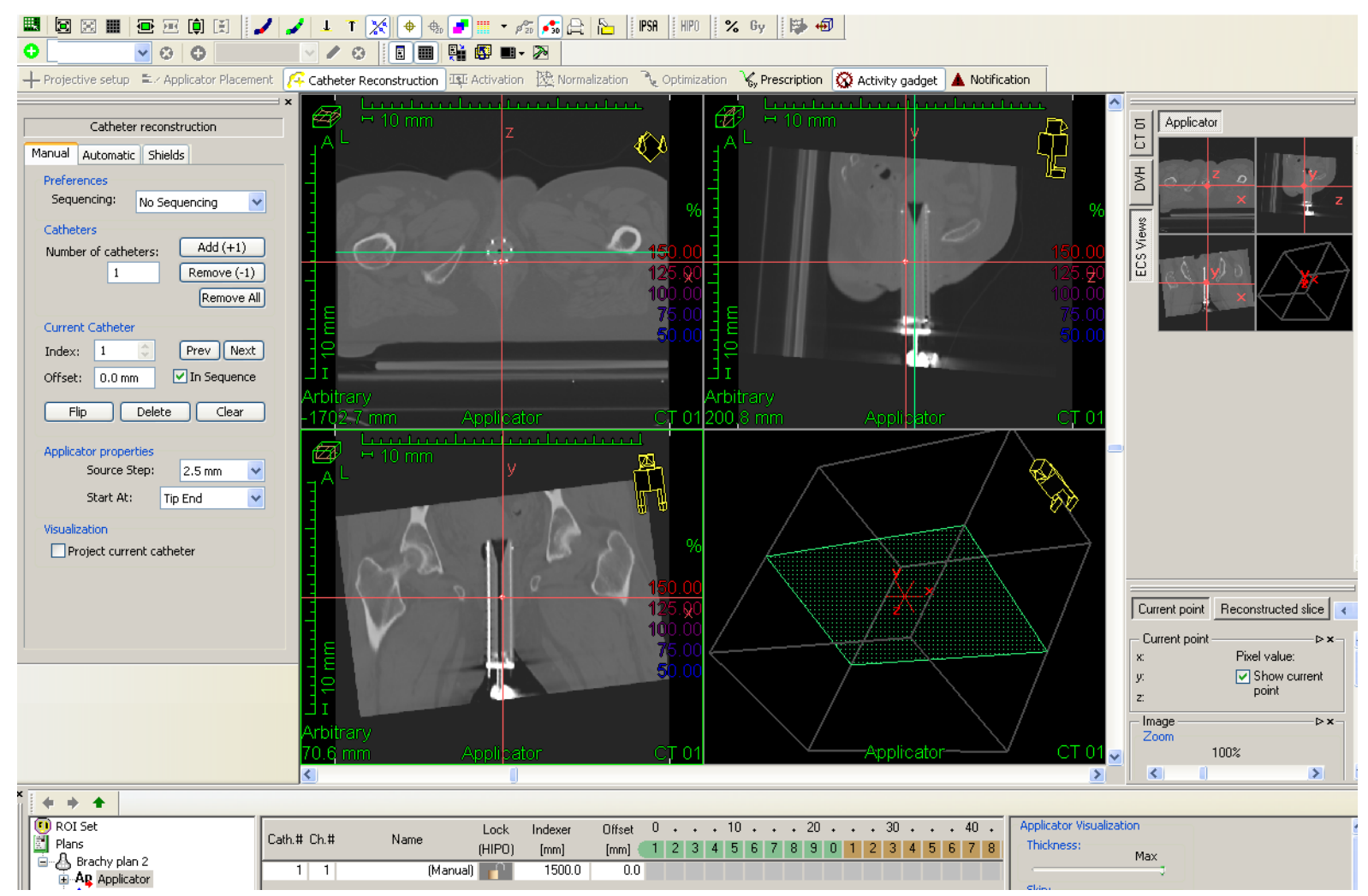

Figure 1. Aligning TPS axis to the center of applicator. 
in the surrounding catheters, though the time at each dwelling positions are often optimized. The advantages of the multi-channel design for cylindrical applicator was recognized in the later time [2] [3], and until recent a step-by-step treatment methodology with multi-channel (Miami) applicators was reported following the guideline of American Brachytherapy Society [4]. Nevertheless, the original purpose for multi-channel applicator was for treatments of cervical cancer rather than vaginal cancers [1]. However, with recent utilization of image-guidance, i.e. a same day MRI acquisition registered to the planning CT, the use of multi-channel applicators meets a significant increase because asymmetrical prescriptions to vaginal wall become more common, presenting novel topics for medical physicists in planning such HDR procedures.

HDR brachytherapy with multiple independent catheters has been mature for many years. The most typical cases would be a) sarcoma treated with HDR brachytherapy of multiple catheters, and b) accelerated partial breast irradiation (APBI) with brachytherapy. Due to the complexity of planning, these cases typically require significant treatment planning time, and the first treatment is typically delivered on the following day. However, for image-guided vaginal cases with multi-channel applicators, the whole procedure is required to be completed on the same day even though an extra MRI scan is added as well as much more complicated planning is also expected. It is fairly challenging to create a timely plan with precision, especially in medical centers of high patient volume. For this reason, novel treatment planning techniques are highly expected and demanded for planners to significantly shorten planning time and reduce potential for errors in the timely planning procedure.

\section{Materials and Methods}

\subsection{Traditional Planning for Typical Single-Catheter GYN Cylinder}

Treatment planning for single-catheter GYN cylindrical applicator has become standardized and mature in HDR brachytherapy. For instance, a typical prescription is to deliver a fractional dose (5 - $10 \mathrm{~Gy}$ ) to vaginal surface or to a certain depth (e.g. $5 \mathrm{~mm}$ ) for 3 - 5 fractions. Regardless the number of treatment fractions, the total dose from HDR treatments is typically less than $30 \mathrm{~Gy}$.

As usual, a typical HDR brachytherapy for GYN cylindrical applicator planning include the following steps: a) contouring all structures; b) digitizing all catheters; c) applying virtual dwelling positions in each catheter; d) generating prescription points or volume; e) prescribing doses and fractionations; f) dose normalizing, g) optimization and review. In the whole procedure of planning, all steps could be considered standardized except for step d), since the treatment planning system (TPS) provides multiple tools to generating the prescriptions. In GYN vaginal cylinder cases, the prescription is typically to point arrays (e.g. an array of points at $5 \mathrm{~mm}$ depth in vaginal wall, parallel to the catheter) rather than volumes.

Based on our clinical experiences, the most convenient way to achieve timely planning with good precision is to align the planning coordinate system (i.e. z-axis) along the applicator-defined coordinates system (i.e. axis of catheter), and then directly generate a prescription point array at a certain depth of vaginal wall parallel to the catheter axis, by utilizing the TPS to define points radially from the central catheter (the distance should be prescription depth + radius of applicator). In this method, each prescription point in the point array is corresponding to a dwelling point in the catheter with the same fixed distance, though the number of points in the array is adjustable.

Clinically, if the cylindrical applicator is properly selected to fit the patient, prescription points at vaginal surface are deemed identical to the applicator surface. Though multiple arrays of points could be generated accordingly, this option is virtually unnecessary because the dose is always symmetrically distributed around the applicator for single catheter treatments.

Given the method above, it is our experience that the time to plan for single-catheter GYN vaginal cylinder cases, excluding the contouring time, is typically 3 - 4 minutes for seasoned medical physicists. For busy radiation oncology centers with serial HDR brachytherapy cases daily, this method ensures precision of efficient planning.

A frequently performed alternate method is to visualize the vaginal wall or depth from the CT slices, and then manually generate the points one-by-one. Another method is to generate the volume of the cylindrical applicator, and then extract some points from the applicator volume. These methods are complicated, both introducing uncertainties in creating prescription points in addition to taking longer to define. We highly recommend the axis-point method since it minimizes the uncertainty and reduces planning time. 


\subsection{Novel Planning for IGBT with Multi-Catheter GYN Cylinder}

For vaginal case of IG-HDR brachytherapy, a gross tumor volume (GTV) can be contoured based on the MRI information, and this volume is then transferred to the planning CT through the MRI/CT image registration. Therefore, a typical prescription for IG-HDR vaginal cases is an array of points at the disease side of vaginal wall, parallel to the applicator axis, plus a clinical treatment volume (CTV) beyond this point array to be covered by the prescription dose.

From the above review for single-catheter applicator planning, the axis-point method is convenient. However in the multi-channel applicator, the center of the applicator is typically hollow with no catheter, thus the axispoint method above will not work because there are no dwelling points in the center. We propose the pseudo central-dwelling (PCD) method to resolve the difficulties in implementing the convenient axis-point approach. All figures listed below are extracted from Nucletron ${ }^{\mathrm{TM}}$ Oncentra TPS (v4.3).

A typical vaginal case of IG-HDR brachytherapy is shown in Figure 1. The patient was diagnosed early stage vaginal carcinomas. The disease can be visualized with sufficient contrast on the MRI scan and hence was contoured on the right side of vagina. The MRI was then registered to the planning CT of typical $2.0 \mathrm{~mm}$ slice thickness, and the contoured structures were copied to the CT for planning with Nucletron ${ }^{\mathrm{TM}}$ Oncentra TPS. The cylindrical multi-channel applicator has 6 catheter channels distributed evenly, and the center is hollow path with no catheter.

The initial setup parameters (step size $2.5 \mathrm{~mm}$, starting from tip-end etc.) are illustrated in Figure 1. The TPS coordinate system (illustrated by ECS view) is first aligned to the center of the multi-channel applicator, in which the center is a hollow path.

Since the left side of the vagina should be spared as the early-stage disease is only at right side, the source should not dwell in the catheters at the left side of applicator, and hence those catheters may not be digitized. In this case, only the 3 catheters at the right side are digitized, marked as Upper-right, Middle-right and Lowerright. Or, to be more precise, planners may use Anterior-right, Right and Posterior-right to name those catheters.

To be efficient in planning, the central hollow path of the applicator is also digitized, as a pseudo-catheter. The digitization is straight-forward since the hollow path is a straight line, thus no markers are needed. The digitized hollow path can be numbered as the $4^{\text {th }}$ catheter with an assigned name "Central”, as illustrated in Figure 2. Please note, as the multi-channel applicator is not standardized, the lengths measured by the source position si-

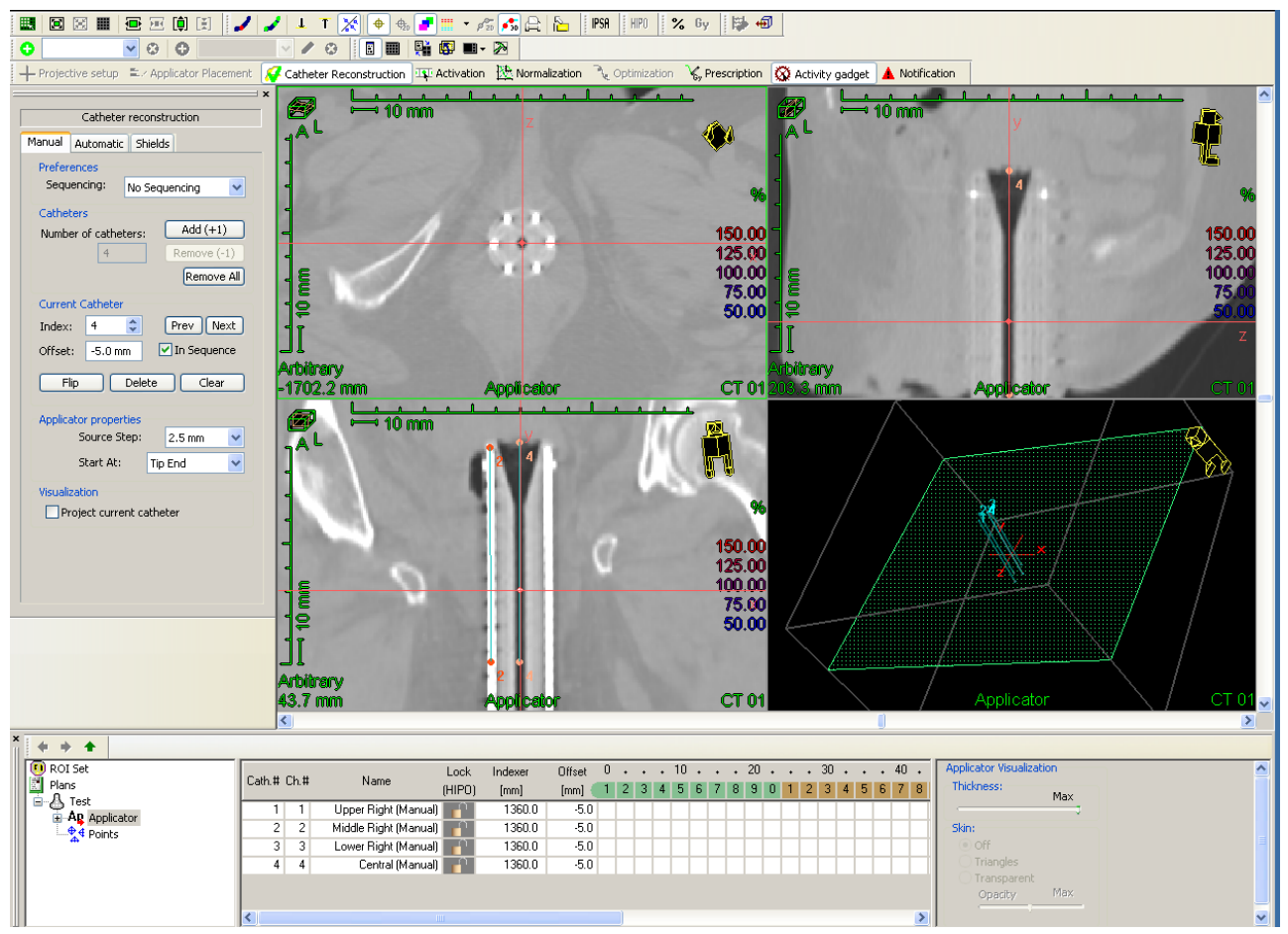

Figure 2. Digitizing all catheters at right side of vagina and the pseudo-catheter at central hollow path. 
mulator (SPS) are not $1500 \mathrm{~mm}$, as indicated in the figure. The non-zero offsets are also due to this non-standardization.

All digitized catheters are then activated with the given step-size of source dwelling (e.g. $2.5 \mathrm{~mm}$ in this case). The pseudo catheter is also activated, as if it is a normal catheter, as shown in Figure 3. This is necessary because the prescription points are based on the pseudo dwelling positions in the pseudo catheter.

For these multi-channel cylinders the prescription is typically on one side of vagina, either at vaginal surface or at certain depth (e.g. $5 \mathrm{~mm}$ depth), such that the other side of vaginal wall can be spared. Though there are multiple ways to generate the prescription point array our experiences favor the axis-points approach. To achieve this, use the dwelling points in the pseudo catheter as references (by choosing the "Axis points" in TPS), and assigning the distance from the array to the axis to be the radius of the applicator. In Figure 4, the prescription points array is named as RT_Cylinder_Surface. As needed in the prescription, another reference point array at certain depth (e.g. $5 \mathrm{~mm}$ in the illustrated case) can also be generated similarly, as illustrated in Figure 5 .

The final step is to delete the pseudo dwelling points after the prescription points were generated, since the pseudo catheter does not even exist and hence will not be used for treatment. After deleting all the dwelling points in the pseudo catheter, the remaining dwelling points as well as the iso-dose lines (IDLs) are real, as illustrated in Figure 6. The IDLs then can be normalized to the prescription points (Figure 7), and the optimization could be performed in later steps.

Comparing to other planning techniques to generating prescription points, the pseudo-catheter method achieves quality, robust and timely HDR plans with minimal uncertainties, primarily due to the simplicity of axis-point approach as well as the pseudo dwelling points technique.

\section{Discussions}

The major advantages for HDR brachytherapy over low dose-rate (LDR) brachytherapy have been: 1) HDR is

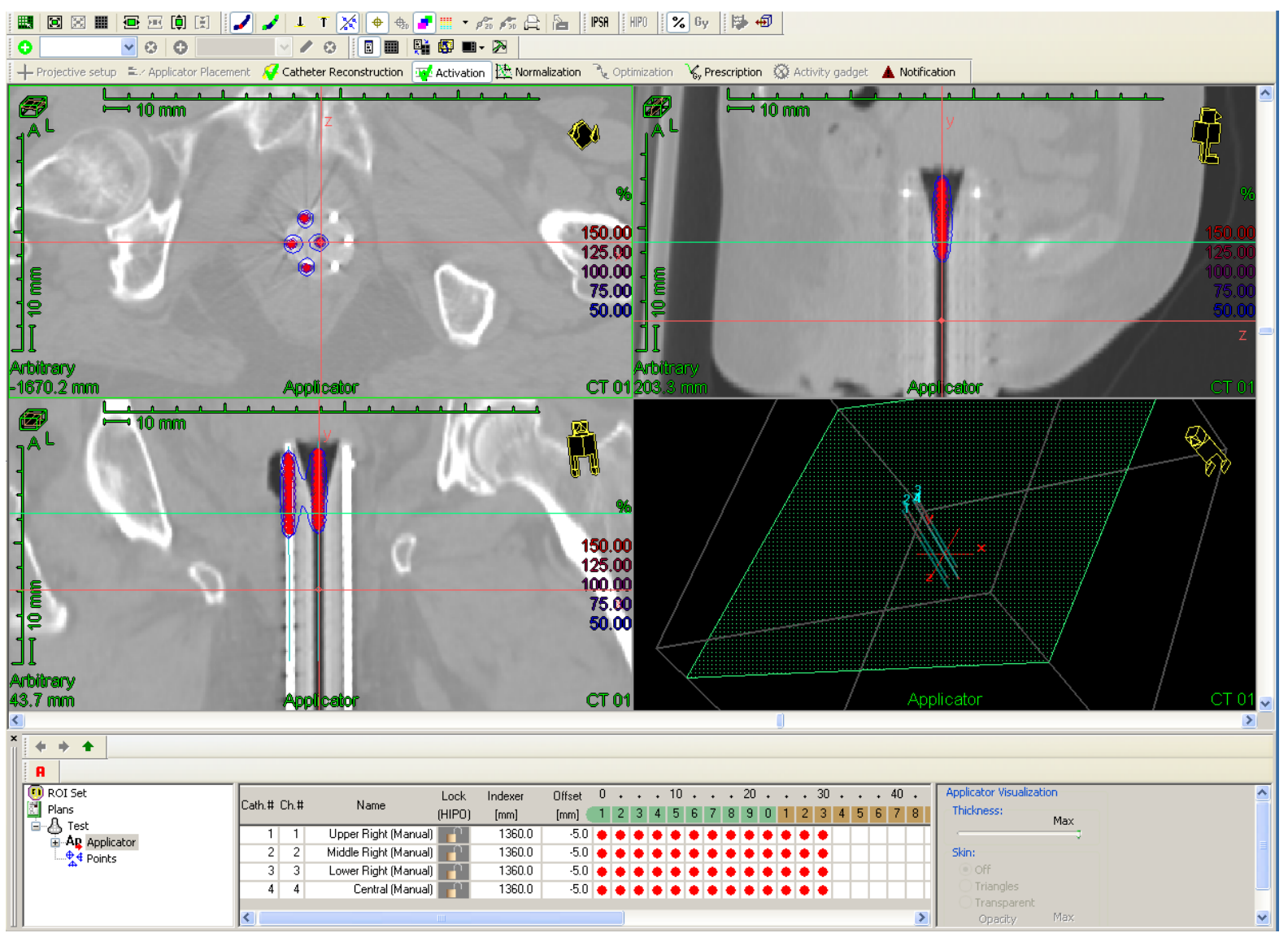

Figure 3. Activating all catheters, including the pseudo-catheter. 


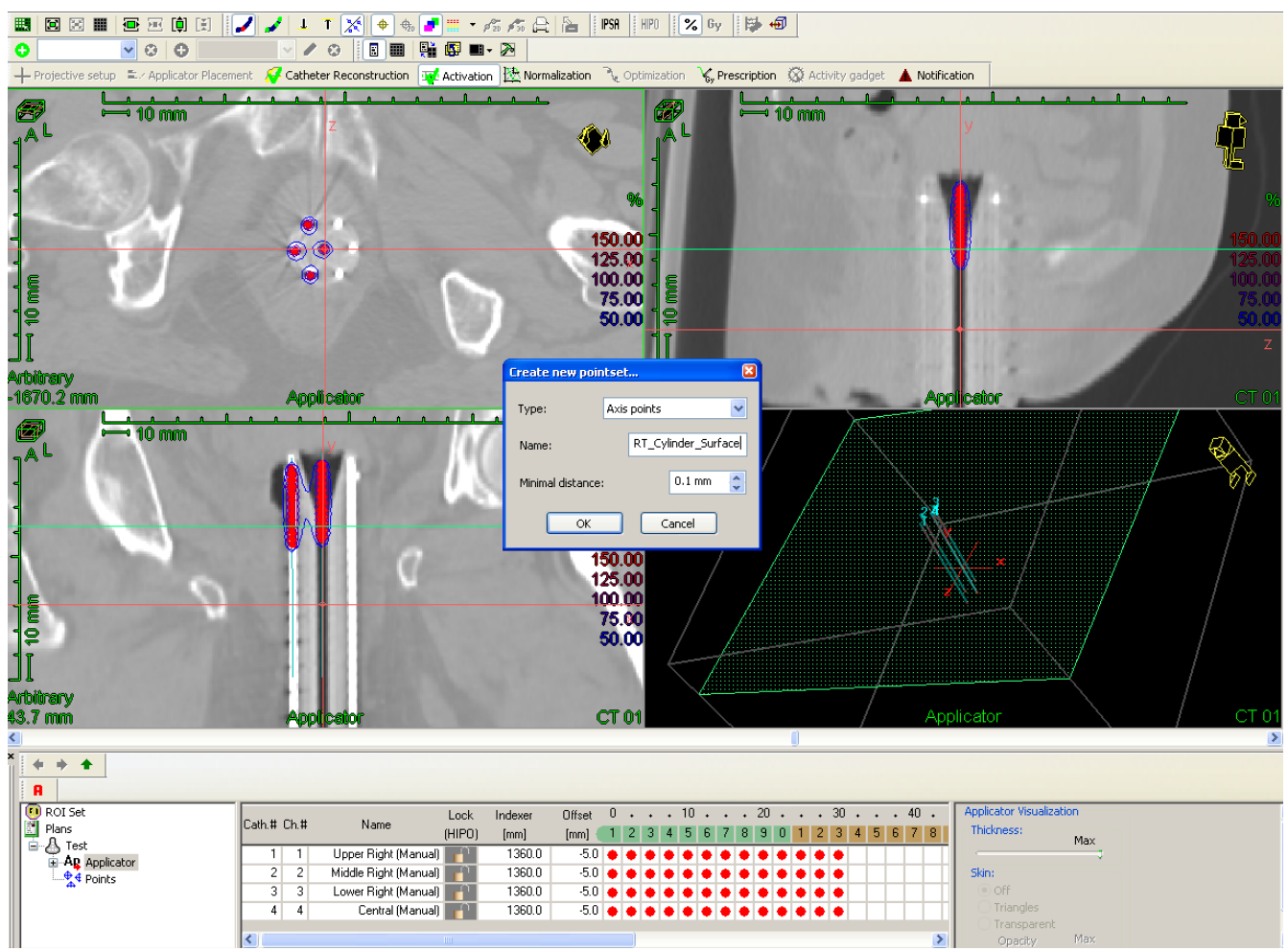

Figure 4. Adding prescription points with axis-point method.

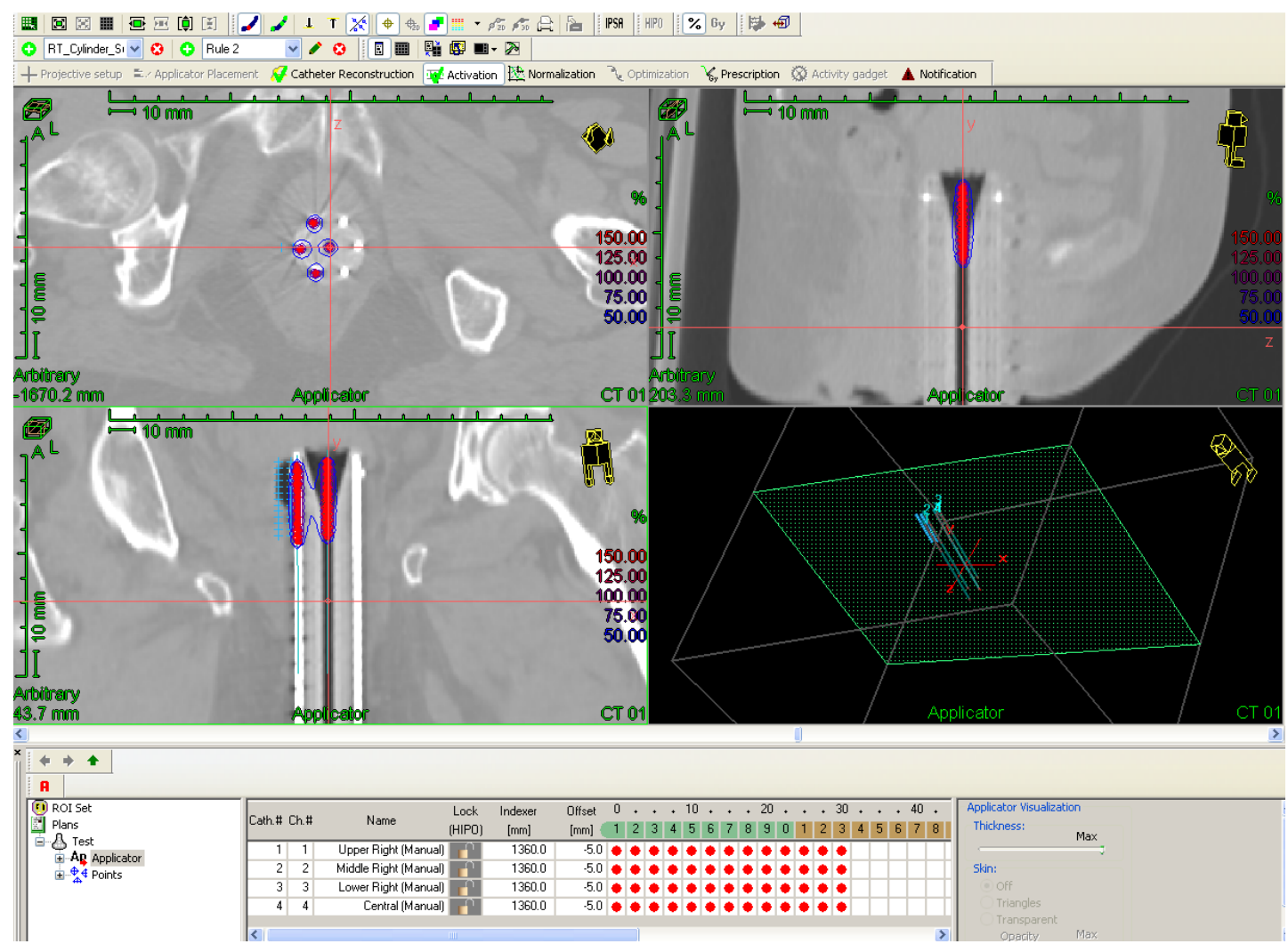

Figure 5. Two arrays of prescription points are generated based on pseudo catheter, as shown on the lower-left panel of the figure. 


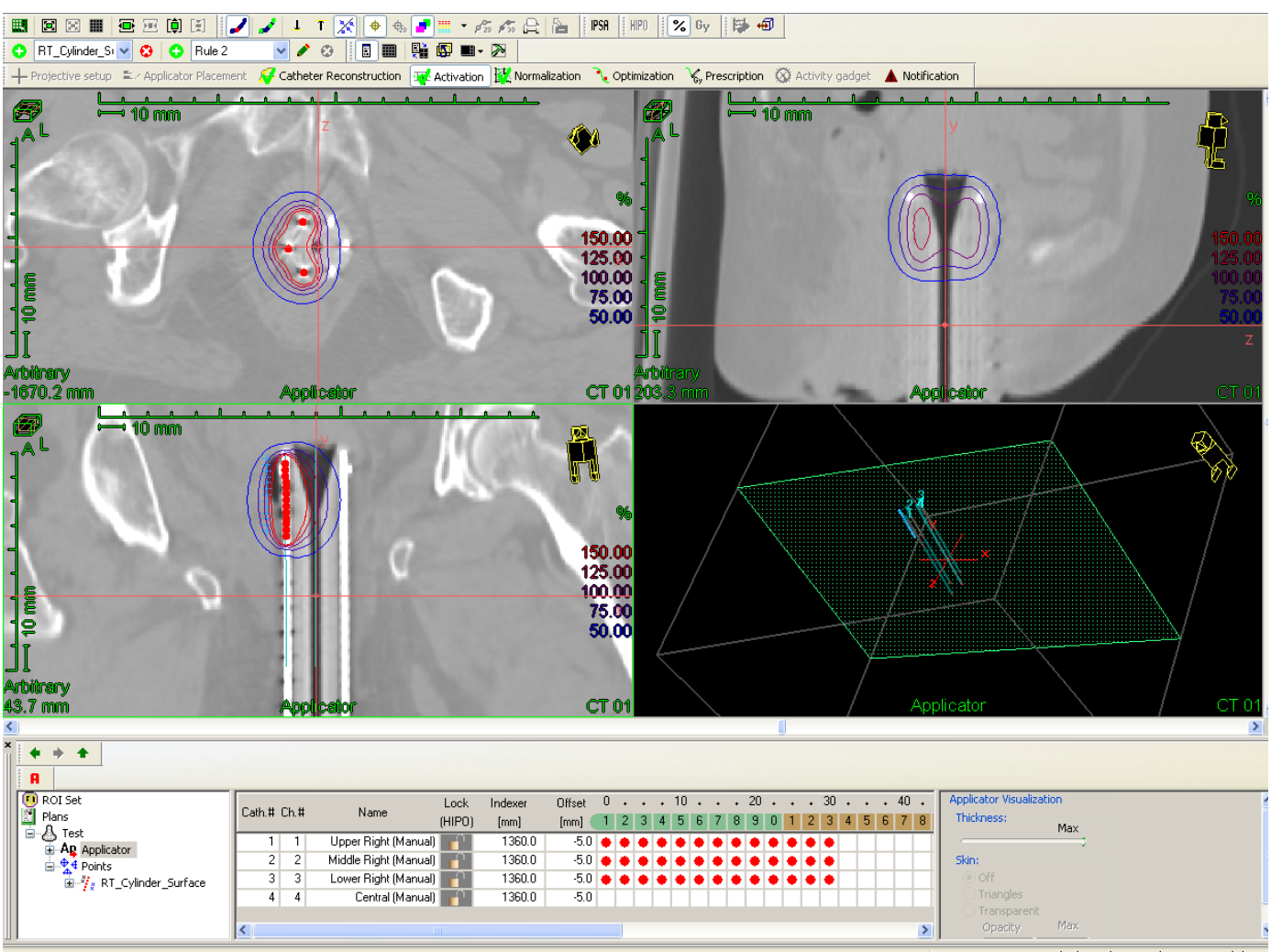

Figure 6. Removing all dwelling points in pseudo catheter (channel 4), the IDLs become real.

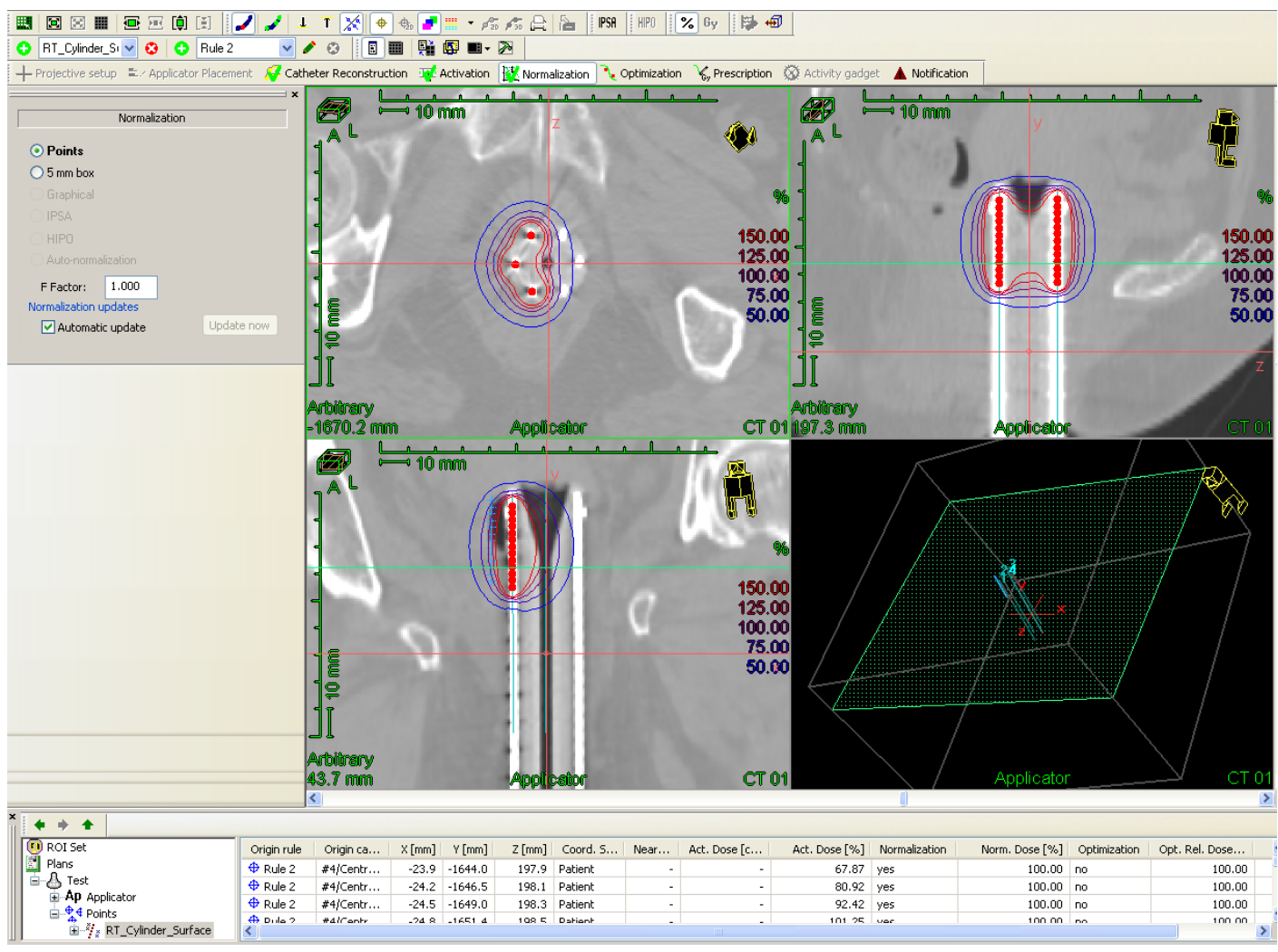

Figure 7. Normalizing the dose to prescription points. 
applicable to outpatients; and 2) a typical HDR procedure is short in time-it only takes a few hours, which including a) applicator installation and patient recovery; b) CT scan or orthogonal films; c) planning; d) dose delivery and e) applicator removal. Compare to the lengthy LDR treatment procedure which typically takes several days for inpatients, those advantages are extremely significant. However, with the application of image-guidance, IG-HDR has weakened those advantages to some extent, because an extra MRI scan is added to the HDR procedure with corresponding image registration, and the asymmetrical prescription become more difficult to plan, which make the whole procedure lengthy and intolerable by patients. For this reason, IG-HDR would be less applicable than it was expected.

The solution for the issues in prevalent IG-HDR may focus on 2 parts. First, the CT scan could be removed from the whole procedure since an MRI scan alone is sufficient for target \& critical organ delineations; in addition, the CT numbers are useless in dose calculations because water-equivalent approximation is always taken in brachytherapy treatment planning system. In another word, an MRI-based procedure would be more applicable to prevalent IG-HDR. However, this concern is out of the scope of this study. The second option is to significantly shrink the planning time without causing medical errors in typical IG-HDR procedure, even though asymmetrical prescriptions demand more complicated planning. The ideal planning time for IG-HDR procedures should be the same or at least compatible to that in typical GYN cases with vaginal cylinder applicators. The results of our study, though primitive, seem to fulfill this approach with good significance.

\section{Conclusions}

IG-HDR (or IGBT) brachytherapy utilizing multi-channel (Miami) applicator has been increasingly implemented in recent years, mainly for early stage vaginal carcinomas. The purpose of this approach is to deliver dose more precisely to the disease such that the healthy part of vagina wall can be spared. To achieve this, the patient needs an extra MRI scan in the morning to delineate the areas to be treated, preferably the scan is acquired with a MRI compatible HDR applicator in place to ensure accurate registration between the MRI and the planning CT. The planning CT is typically collected shortly after the MRI to ensure the precision of image registration. Due to the complexity of planning asymmetrical proscription, as well as the extra MRI scan, the whole IG-HDR procedure becomes lengthy and less tolerable by patients, which has weaken the major advantages of HDR over LDR brachytherapy, and hence potentially significantly reduced the applicability of IG-HDR techniques. For this reason, novel planning techniques for IG-HDR planning is highly demanded.

Planning for IG-HDR cases utilizing multi-channel applicators is novel and challenging to medical physicists involved in the typical HDR brachytherapy procedures. The planning procedure involving in asymmetrical prescription has been difficult to execute with good efficiency and minimal uncertainties. Based on extensive experience on single-catheter and multi-channel applicator for HDR procedures, we propose the step-by-step pseudo central-catheter method in generating point arrays for asymmetrical prescription, which robustly achieves quality treatment plans with minimal uncertainties in a timely manner.

\section{References}

[1] Houdek, P.V., Schwade, J.G., Abitbol, A.A., Pisciotta, V., Wu, X.D., Serago, C.F., Markoe, A.M., Levin, A.A., Averette, H.E., Aevin, B.U., et al. (1991) Optimization of High Dose-Rate Cervix Brachytherapy; Part I: Dose Distribution. International Journal of Radiation Oncology*Biology* Physics, 21, 1621-1625. http://dx.doi.org/10.1016/0360-3016(91)90341-Z

[2] Demanes, D.J., Rege, S., Rodriquez, R.R., Schutz, K.L., Altieri, G.A. and Wong, T. (1999) The Use and Advantages of a Multichannel Vaginal Cylinder in High-Dose-Rate Brachytherapy. International Journal of Radiation Oncology*Biology* Physics, 44, 211-219. http://dx.doi.org/10.1016/S0360-3016(98)00453-2

[3] Tanderup, K. and Lindegaard, J.C. (2004) Multi-Channelintracavitary Vaginal Brachytherapy Using Three-Dimensional Optimization of Source Geometry. Radiotherapy \& Oncology, 70, 81-85. http://dx.doi.org/10.1016/j.radonc.2003.11.006

[4] Iftimia, I., Cirino, E.T., Mower, H.W. and McKee, A.B. (2013) Treatment Planning Methodology for the Miami Multichannel Applicator Following the American Brachytherapy Society Recently Published Guidelines: The Lahey Clinic Experience. Journal of Applied Clinical Medical Physics, 14, 4098. 